\title{
Le premier témoin manuscrit des Actes de Pilate (ÖNB 563) : antiquité et autorité de la traduction latine d'un texte grec ${ }^{1}$
}

Le texte connu sous le titre moderne d'Actes de Pilate est un récit qui naît peut-être au $\mathrm{II}^{\mathrm{e}}$ siècle, dans un milieu judéo-chrétien, mais prend une forme proche de sa forme actuelle sans doute au cours du IV ${ }^{\mathrm{e}}$ siècle. Il reflète différentes polémiques des premiers siècles du christianisme sur la personne de Jésus, l'attitude des juifs, et la résurrection. Il se compose à mon sens de deux moments principaux : le procès de Jésus, narré avec force détails accusations diverses, comparution des témoins que sont les miraculés (le paralytique, la femme hémorroïsse...), sentence de Pilate; et l'histoire de Joseph d'Arimathie, qui commence par son incarcération après la crucifixion, si bien que le premier acte du ressuscité est de libérer Joseph de prison, faisant de lui le messager de sa résurrection auprès des juifs. À une époque plus tardive, entre le $\mathrm{VI}^{\mathrm{e}}$ et le $\mathrm{IX}^{\mathrm{e}}$ siècle, a été ajouté à ce texte le récit de la Descente aux Enfers, et c'est l'ensemble Actes de Pilate et Descente aux Enfers que 1'on appelle aujourd'hui, dans le milieu scientifique, l'Évangile de Nicodème - de son titre médiéval. Ce texte a en effet connu une grande popularité au Moyen Âge comme à l'époque moderne.

Composés en grec, les Actes de Pilate ont été traduits très tôt en copte, en syriaque, en christo-palestinien, en arménien, en géorgien, et en latin ${ }^{2}$ - tout cela avant l'addition de la Descente aux Enfers. L'étude des Actes de Pilate, et l'édition préparée en ce moment pour le Corpus christianorum, Series apocryphorum, par des membres de l'Association pour l'étude de la littérature apocryphe chrétienne ${ }^{3}$, prend donc pour texte de base les formes grecques les manuscrits se répartissent en deux familles, $\varphi$ et $\chi$, auquels s'ajoutent des témoins inclassables ${ }^{4}$. Chaque leçon et chaque variante sont comparées avec tous les témoins linguistiques : leur coïncidence a des chances de refléter une forme ancienne du texte.

\footnotetext{
${ }^{1}$ Cet article est issu du remaniement d'une communication donnée en février 2016 devant la Société des études grecques, dont je remercie les membres pour leurs remarques. Pour la préparation de cette étude, j'ai grandement bénéficié des conditions de travail et des richesses de la bibliothèque de la fondation Dumbarton Oaks (Washington, D.C.) et j'exprime toute ma reconnaissance à cette institution.

${ }^{2}$ Pour des précisions sur l'époque de ces traductions, qui s'échelonnent vraisemblablement du V ${ }^{\mathrm{e}}$ au VII ${ }^{\mathrm{e}}$ siècle, et sur leur circulation, voir J.-D. DuBOIS, «L'utilisation des Actes de Pilate au IVe siècle », Apocrypha 2 (1991), p. 85-98, p. 94-96 ; J.-D. DuBOIS et Z. IZYDORCZYK, « Nicodemus's Gospel before and beyond the Medieval West », dans The Medieval Gospel of Nicodemus. Texts, Intertexts, and Contexts in Western Europe, Tempe (AZ), 1997, p. 21-42, p. 29-40, ainsi que les diverses contributions des journées d'études sur les Actes de Pilate/ Évangile de Nicodème qui se tinrent à Winnipeg en 2010, parues dans Apocrypha 21 (2010).

${ }^{3}$ L'équipe chargée de la nouvelle édition des Actes de Pilate rassemble, sous la responsabilité de J.-D. Dubois, A. Frey, C. Furrer, R. Gounelle, J. Haynes, Z. Izydorczyk, B. Outtier, J.-N. Pérès, G. Roquet, S. Torres Prieto et moi-même. Le présent travail bénéficie largement de l'accès au texte en cours d'édition et de la science des membres du groupe ; qu'ils trouvent ici l'expression de ma gratitude pour leur générosité. Jusqu'à la parution de cette édition, le texte de référence reste celui que l'on trouve dans K. VON TISCHENDORF, Evangelia apocrypha, Leipzig, $1853,{ }^{2} 1876$.

${ }^{4}$ Les manuscrits grecs, leur référence et leur classement en famille sont exposés dans C. FURRER, «La recension greque ancienne des Actes de Pilate », Apocrypha 21 (2010), p. 11-30 et, pour trois manuscrits découverts par la suite, dans C. FURRER et C. GUIGNARD, "Titre et prologue des Actes de Pilate: nouvelle lecture à partir d'une reconstitution d'un état ancien du texte », Apocrypha 24 (2013), p. 139-206, Appendice 4, p. 204-205.
} 
Le témoin manuscrit le plus ancien des Actes de Pilate, toutes langues confondues, est un palimpseste latin. Ce manuscrit de la bibliothèque de Vienne (Österreichische Nationalbibliothek, Cod. 563) transmet des écrits patristiques dans l'écriture supérieure $\left(\mathrm{IX}^{\mathrm{e}} \text { siècle }\right)^{5}$. Il est issu du démembrement d'un parchemin qui rassemblait au moins trois textes, les Actes de Pilate, l'évangile canonique de Matthieu (dont sont conservés des fragments des chapitres 26 à 28) ${ }^{6}$ et l'Évangile de l'enfance de Jésus. L'histoire de la redécouverte de ce manuscrit à l'époque moderne a été présentée par Guy Philippart, qui a aussi donné la transcription diplomatique de ce qui était lisible ${ }^{7}$.

Pour l'écriture inférieure, l'étude codicologique et paléographique la plus poussée sur le palimpseste de Vienne ${ }^{8}$ propose une datation au $\mathrm{V}^{\mathrm{e}}$ siècle$^{9}$. Pour les Actes de Pilate, qui seuls retiendront l'attention ici, ce témoin latin est ainsi antérieur de six siècles au moins au plus ancien manuscrit grec. Il peut mériter une attention particulière dans deux contextes : à l'intérieur de la tradition latine, pour évaluer l'originalité du texte qu'il transmet; et par rapport au grec et éventuellement aux versions orientales, pour faire l'hypothèse du texte source de la traduction. L'évaluation à l'intérieur de la tradition latine cherche à situer le palimpseste parmi les trois grandes familles qui ont été distinguées et nommées $\mathrm{A}, \mathrm{B}$ et $\mathrm{C}-\mathrm{la}$ famille $\mathrm{A}$ est la majoritaire, la famille $\mathrm{C}$ dépend de celle-ci, la famille $\mathrm{B}$ est peut-être issue d'une révision d'un texte de la famille A sur le grec - et par rapport à des manuscrits isolés qui entretiennent parfois une grande proximité avec le palimpseste ${ }^{10}$. Mais c'est surtout la seconde perspective qui est l'objet de cet article : il s'agit d'analyser des données que nous avons mises au jour en préparant un commentaire suivi, mot à mot, du texte des Actes de Pilate dans le palimpseste de Vienne, à l'occasion duquel nous avons proposé une rétroversion expérimentale du latin en $\operatorname{grec}^{11}$. La question est de savoir ce que la traduction

\footnotetext{
${ }^{5}$ E.A. Lowe, Codices Latini Antiquiores. A Palaeographical Guide to Latin Manuscripts Prior to the Ninth Century, t. 10 : Austria, Belgium, Czechoslovakia, Denmark, Egypt, and Holland, Oxford, 1963, § 1485, p. 14, repris par Z. IZYDORCZYK, Manuscripts of the Evangelium Nicodemi. A Census, coll. "Subsidia Mediaevalia » 21, Toronto, 1993, § 393, p. 192.

${ }^{6}$ Cf. A.-C. BAudoin, « La Vetus Latina de Mt 26-28 dans le manuscrit de Vienne, ÖNB, cod. 563 », à paraître dans la Revue des études augustiniennes.

${ }^{7}$ G. PhilipPART, « Fragments palimpsestes latins du Vindobonensis 563 ( $\mathrm{V}^{\mathrm{e}}$ siècle ?). Évangile selon S. Matthieu, Évangile de l'enfance selon Thomas, Évangile de Nicodème », Analecta Bollandiana 90 (1972), p. 391-441; G. PHILIPPART, « Les fragments palimpsestes de l'Évangile de Nicodème dans le Vindobonensis 563 ( $\mathrm{V}^{\mathrm{e}}$ siècle ?)», Analecta Bollandiana 107 (1989), p. 171-188. Le titre d'Histoire de l'enfance de Jésus est aujourd'hui préféré à celui d'Évangile de l'enfance selon Thomas, pour éviter la confusion avec l'Évangile de Thomas de Nag Hammadi. Cf. S. VoICU, "Notes sur l'histoire du texte de l'Histoire de l'enfance de Jésus », Apocrypha 2 (1991), p. 119-132, p. 122.

8 Notice et bibliographie sont disponibles sur le site de l'Österreichische Nationalbibliothek (http://data.onb.ac.at/rec/AL00173440). Pour les fragments de Matthieu, cf. R. GRYSON, Altlateinische Handschriften - Manuscrits vieux latins. Répertoire descriptif. Première partie : Mss 1-275; d'après un manuscrit inachevé de Hermann Josef Frede †, coll. « Vetus Latina. Die Reste der altlateinischen Bibel»1/2A, Fribourg-en-Brisgau, 1999, p. 67 (manuscrit 43).

9. M. DESPINEUX, «Une version latine palimpseste du v siècle de l'Évangile de Nicodème (Vienne, ÖNB MS 563), Scriptorium 42 (1988), p. 176-183.

${ }^{10}$ Ces manuscrits seront désignés par le numéro qui leur a été attribué par Z. IZYDORCZYK, Manuscripts of the Evangelium Nicodemi. A Census, coll. «Subsidia Mediaevalia»21, Toronto, 1993, où l'on trouvera les références complètes (liste des manuscrits latins mentionnés dans la présente étude en annexe de cet article).

${ }^{11}$ A.-C. BAudoin et Z. IZYDORCZYK (éd.), The Oldest Manuscript of the Acts of Pilate: A Collaborative Commentary on the Vienna Palimpsest, Proceedings of the Second International Summer School on Christian Apocryphal Literature (ISCAL), Strasbourg, 9-12 June 2014, à paraître en format électronique, Université de
} 
latine du plus ancien témoin des Actes de Pilate peut nous apprendre sur son original grec et comment on peut évaluer le texte grec présent derrière la traduction.

\section{Une traduction parfois servile à l'extrême}

La valeur que le palimpseste possède de fait par son antiquité n'a d'intérêt pour nous que si la traduction latine reflète avec exactitude le texte grec ; or, grâce à la multiplicité des témoins dont on dispose pour les Actes de Pilate, on peut montrer que le traducteur a été fidèle au grec au point de commettre des hellénismes de différentes natures.

Parmi les hellénismes de vocabulaire caractéristiques du palimpseste et que l'on ne retrouve pas dans l'ensemble de la tradition latine, on peut relever tout d'abord les

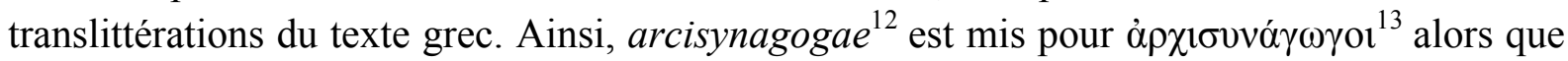
les manuscrits latins - des Actes de Pilate comme des évangiles - ont habituellement principes sacerdotum. Dans la citation de Lc 2,35, dans les mots adressés par Syméon à Marie lors de la présentation de Jésus au temple, «Et toi aussi une épée te transpercera le cœur »,

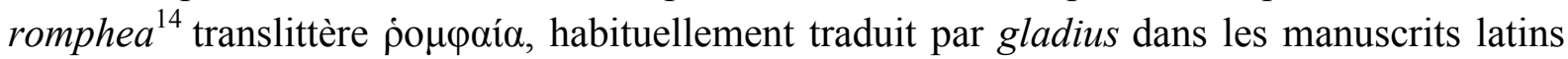
de Luc $^{15}$.

Un cas spécial de translittération peut être l'importation des noms propres. La plupart ne présentent rien de significatif ; on peut toutefois noter que le traducteur ou le copiste du manuscrit latin avait le souci de garder les formes grecques: Aijó $\pi \tau$ ov est transcrit avec Aeg $[y p] t^{16}{ }^{(1 ' i n i t a l e ~ e n ~ a e ~ e s t ~ d o n c ~ a t t e s t e ́ e) ~ e t ~}[\mathrm{Aeg}] \mathrm{y}[\mathrm{p}]<t o>^{17}$ (le $y$ est donc attesté) ; le $\varphi$ du nom de Caïphe est transcrit par $p h$. Ce n'est toutefois pas systématique : on a par exemple Simeone ${ }^{18}$ pour $\Sigma v \mu \varepsilon \tilde{\omega} v o s$. Dans une occasion, la flexion semble calquée sur le grec : Caipha semble être utilisé comme génitif de Caiphas ${ }^{19}$ (grec Kaïápa).

Pour le lexique, le traducteur n'applique pas ses choix de manière systématique : un mot ou une expression ne sont pas toujours traduits de la même façon, pour autant que l'on puisse en faire l'hypothèse à partir des témoins grecs. Ainsi, la conjonction de subordination

Strasbourg. Je remercie Z. Izydorczyk pour sa grande générosité dans le partage de son travail et le don de son temps.

${ }^{12}$ Seg. XVI, ch. 16.1.1, J2(167v), cf. seg. XV, ch. 14.1, I3(168r) et seg. XVIII, ch. 16.3.2, K7(170r). Les indications sont le numéro de segment dans la transcription de Philippart, celui du chapitre - dans la numérotation adoptée dans R. GOUNELLE et Z. IZYDORCZYK, L'Évangile de Nicodème ou les Actes faits sous Ponce Pilate (recension latine A), coll. «Apocryphes » 9, [s.l.], 1997 -, la référence de la page dans la recomposition ordonnée du palimpseste, et le numéro du folio dans le manuscrit actuel.

${ }^{13}$ Pour le grec je cite, sauf précision contraire, le texte de l'édition en préparation pour le Corpus Christianorum, series Apocryphorum (famille $\varphi$ ).

${ }^{14}$ Seg. XVII, ch. 16.1.2, J5(125r).

${ }^{15}$ Cf. A. JÜLICHER, Itala. Das neue Testament in altlateinischer Überlieferung, t. 3 : Lucas' Evangelium, Berlin, 1954, ad loc.

${ }^{16}$ Seg. X, ch. 7.2, F2(146v)-F3(153r).

${ }^{17}$ Seg. X, ch. 7.2, F3(153r).

${ }^{18}$ Seg. XVIII, ch. 16.3.2, K3(130r).

${ }^{19}$ Seg. II, prol., B2(152v). Le cas n'est pas certain car la syntaxe est chaotique : on lit sub principatus a

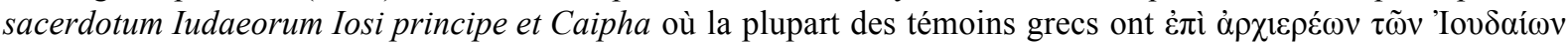

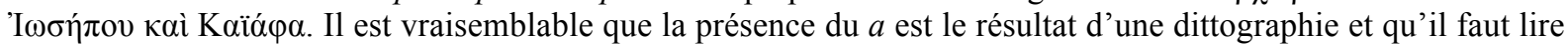

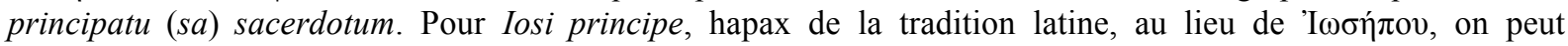
supposer que la dernière syllabe avait été prise, en latin, pour une abréviation de principe, ce qui expliquerait aussi le cas incongru de ce terme. 
ö $\tau$ est traduite par quoniam cinq fois, des chapitres 1.2 à 3.2, puis par quia dans douze occurrences, des chapitres 4.5 à 16.3.2, sans qu'il y ait de variations de sens évidentes (par exemple, au chapitre 2.6, quoniam prend le sens de " parce que », puis de " que » introduisant une complétive). S'il n'était pas isolé, ce trait pourrait révéler un changement d'idiomatisme et peut-être de traducteur, mais l'indice est hélas trop ténu.

Populus traduit généralement $\lambda \alpha$ ós (cinq occurrences au chapitre 16.3.2 et 16.3.3) sauf au chapitre 2.6 où il traduit $\pi \lambda \tilde{\eta} \theta o s$, terme traduit par multitudo aux chapitres $4.5,5.1,6.4$ (quatre occurrences). En revanche, autem semble constamment traduire $\delta \dot{\varepsilon}$ (dix occurrences); le pronom aủ ós, sous ses diverses formes, est toujours traduit par is (quarante-neuf occurrences ; cinq fois, pour des raisons de sens, il est traduit par ipse), sauf une fois, au chapitre 5.1 (illum), et oṽ $0 \varsigma$ par iste (seize occurrences). Peu de conclusions peuvent être tirées de ces pratiques.

Parmi les décalques de structure, on note particulèrement signum contradictum ${ }^{20}$ pour

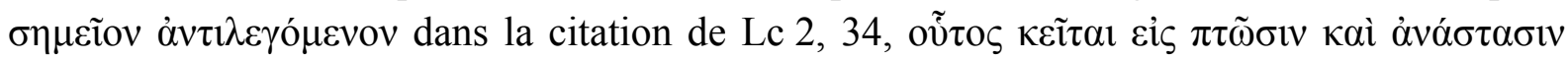

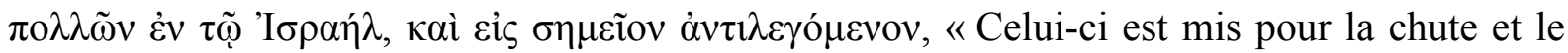
relèvement de beaucoup en Israël, et pour un signe que l'on contradira ». Cette expression obscure est traduite signum cui contradicetur dans la Vulgate et par des expressions proches dans la plupart des Vetus Latina ${ }^{21}$; le participe n'est traduit que par un seul terme dans un manuscrit de la Vetus Latina ${ }^{22}$, contradicentem, et chez Tertullien (De carne Christi 23), contradicibile. Le palimpseste de Vienne est le seul témoin latin à donner à $\alpha \dot{v} \tau \imath \lambda \varepsilon \gamma o ́ \mu \varepsilon v o v$ un sens passif, « un signe contesté ».

Dans le premier chapitre, une des questions posées par Pilate aux juifs est qualiu malarum açtionum ${ }^{23}$ (avec le $-m$ final de qualiu omis ou marqué par un tilde non lisible) : en grec, cette question, Поí $\omega v \kappa \alpha \kappa \tilde{\omega} v \pi \rho \alpha ́ \xi \varepsilon \omega v$, reprend par le génitif les derniers mots des juifs, $\dot{\varepsilon} \theta \varepsilon \rho \alpha ́ \pi \varepsilon v \sigma \varepsilon v$... $\dot{\alpha} \pi$ ò $\kappa \alpha \kappa \tilde{\omega} \nu \pi \rho \alpha ́ \xi \varepsilon \omega v$. Dans le texte latin, en revanche, cette expression a été traduite par çurauit ... a malis actionibus, et le génitif n'a pas lieu d'être dans la question de Pilate. Il s'agit ici d'un calque grammatical.

Le plus bel hellénisme de syntaxe a déjà été relevé par les savants ${ }^{24}$ : Quae est quae agit $^{25}$, question posée par Pilate aux Juifs, ne se comprend que si l'on considère que cette

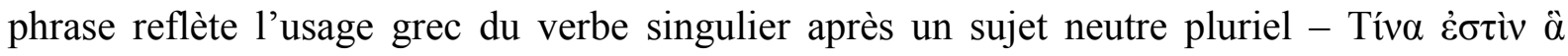
$\pi \rho \alpha ́ \tau \tau \varepsilon 1$; La grande majorité des manuscrits plus tardifs donne à lire Quae sunt quae agit ou Quid est quod agit ${ }^{26}$.

Enfin, dans deux cas, il est possible que des particularités matérielles, liées à l'écriture ou à la disposition de la page, aient eu un effet sur la forme du texte. Au chapitre 16.3.2, dans le contexte assez obscur d'un discours prophétique, on lit dans le palimpseste quid intellegițis. Cette interrogation n'a pas de sens dans la phrase ; elle n'a aucun équivalent dans les

\footnotetext{
${ }^{20}$ Seg. XVIII, ch. 16.3.2, K3-4(130r-v), cf. seg. XVII, ch. 16.1.2, J5(125r).

${ }^{21}$ Cf. A. JÜlICHER, Itala. Das neue Testament in altlateinischer Überlieferung, t. 3 : Lucas' Evangelium, Berlin, 1954, ad loc.

${ }^{22}$ Il s'agit de la traduction latine présente dans le Codex Bezae (Cambridge, University Library, Nn. II. 41, v. 400, traditionnellement désigné par la lettre $d$ ).

${ }^{23}$ Seg. III, ch. 1.1, B5(149r).

${ }^{24} \mathrm{M}$. DespineuX, «Une version latine palimpseste du $\mathrm{v}$ siècle de l'Évangile de Nicodème (Vienne, ÖNB MS 563), Scriptorium 42 (1988), p. 176-183, p. 180.

${ }^{25}$ Seg. III, ch. 1.1, B4(150v).

${ }^{26}$ Seuls certains manuscrits du IX ${ }^{\mathrm{e}}$ siècle gardent cette syntaxe $(133,207,334,158)$.
} 
manuscrits grecs ni dans les versions orientales, qui ont simplement «vous savez»; les manuscrits latins les plus proches ont pour leçon qui intelligitis (129, 213 et 322) ou qui scietis (127 et 129a) qui font de la proposition une relative. Peut-être l'apparition du pronom dans le texte du palimpseste est-il le résultat d'une mélecture du texte grec en onciales : $\Gamma \mathrm{I} \Gamma \mathrm{N} \Omega \Sigma \mathrm{KETE}$, « vous savez », pourrait avoir été lu TI $\Gamma \mathrm{IN} \Omega \Sigma \mathrm{KETE}$, « que savez-vous ? » Ce glissement peut avoir eu lieu dans la copie en grec d'un manuscrit grec ou dans le passage du grec au latin; les copistes des ancêtres des manuscrits inclassables auraient ensuite tenté d'éclairer l'obscurité ainsi conférée à la phrase ${ }^{27}$.

Deuxième situation liée à la présentation matérielle, un possible cas d'insertion d'une glose dans le texte. Lorsque le personnage chargé d'introduire Jésus dans le prétoire étend un linge sous les pieds de celui-ci, l'objet est désigné dans le palimpeste comme facialem inuolu[t]o[r]i[um]. Dans la plupart des manuscrits grecs, le terme est $\kappa \alpha \theta \dot{\alpha} \pi \lambda \omega \mu \alpha$ dans les manuscrits isolés I et $\mathrm{J}$, dans $\varphi^{\mathrm{C}}$ et dans les manuscrits de la famille $\chi$.

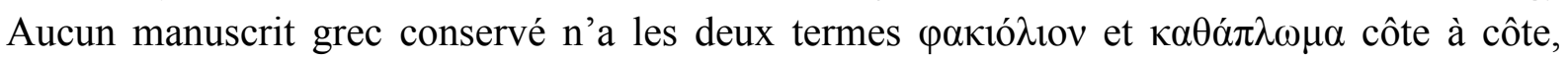
mais l'on pourrait concevoir que le second, de $\kappa \alpha \theta \alpha \pi \lambda$ ó $\omega$, «déplier», soit une glose explicative du premier, qui est la transcription grecque du latin faciale. Le terme inuolutorium serait la traduction du grec $\kappa \alpha \theta \alpha \dot{\pi} \lambda \omega \mu \alpha^{28}$, ce qui laisserait penser que c'est en grec qu'a eu lieu l'association des deux termes. On retrouve l'ensemble facialem inuolutorium dans la famille latine A et dans quelques manuscrits isolés latins $(299,391)$. À ce titre, ces manuscrits latins, au premier rang desquels se trouve le palimpseste, pourraient refléter une forme textuelle qui ne nous est pas parvenue en grec.

\section{L'impossible quête du texte source}

Le texte grec des Actes de Pilate est actuellement édité sur la base de vingt témoins manuscrits datés entre le XII et le XVIII ${ }^{\mathrm{e}}$ siècle. Aucun d'entre eux ne conserve un texte identique à celui qui a pu servir à la première traduction du grec en latin. Toutefois, la lecture des Actes de Pilate dans le palimpseste de Vienne, malgré l'état fragmentaire du texte, n'est pas difficile : on suit parfaitement les différents éléments de l'histoire et il est aisé d'établir une correspondance entre les éléments transmis par le palimpseste et le texte des traditions grecque ou latine. La plupart du temps, on peut penser retrouver le texte grec derrière la traduction latine. L'enjeu serait d'identifier un manuscrit ou une famille de manuscrits qui serait particulièrement proche de la forme du texte grec utilisé comme support pour la traduction latine. Il faut d'emblée confesser que cette quête est infructueuse; quelques passages particulièrement révélateurs montrent la complexité des relations entre les manuscrits. Les passages retenus ici portent uniquement sur des variantes constituées de plusieurs mots ou de plusieurs phrases (omissions, additions, divergences).

\footnotetext{
${ }^{27}$ Dans le texte du palimpseste la phrase se poursuit avec qui[a] retinentia usque in saeculum, termes que l'on retrouve dans le manuscrit 322, et sous des formes proches dans 129 et 213 (127 et 129a: retinenciam). Retinentia semble être le participe présent au neutre pluriel de retineo, une forme qui ne peut être expliquée par

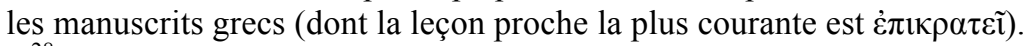

${ }^{28}$ Cette suggestion a été faite par E. VON DOBSchÜTZ, « Der Process Jesu nach den Acta Pilati », Zeitschrift für die Neutestamentliche Wissenschaft und die Kunde des Urchristentums 3 (1902), p. 95, n. 3.
} 
A. Une omission ou une addition partagée avec quelques manuscrits grecs et avec la tradition latine

Quand un passage est absent du palimpseste et d'autres versions du texte, mais présent dans certaines, peut-on estimer s'il s'agit d'une omission - involontaire ou intentionnelle - ou d'une addition postérieure à une première diffusion du texte ?

Au chapitre 4.2, les juifs accusent Jésus de blasphème devant Pilate : Per caesare si quis blasphemauerit dignus est morti. Iste autem aduersus Deum blasphemauit. Cet exposé a tout son sens; seule l'issue du raisonnement a fortiori est laissé en suspens : si celui qui a blasphémé contre César mérite la mort, à plus forte raison celui qui a blasphémé contre Dieu doit être mis à mort. Cette structure se retrouve dans les manuscrits latins des familles $\mathrm{A}$ et $\mathrm{C}$, mais aussi dans les manuscrits grecs $\varphi^{\mathrm{YLZ}}$, et dans les manuscrits grecs inclassables B, et $\mathrm{N}$ (le passage est récrit dans $\chi$ ). En revanche, dans les autres manuscrits grecs, et dans l'arménien, le copte, le géorgien, le syriaque et la famille latine $B$, le texte est plus long : les juifs

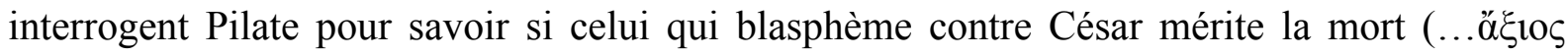

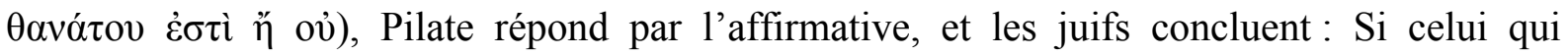

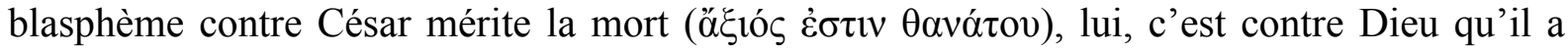
blasphémé. Faut-il considérer qu'il y a eu un saut du même au même assez tôt dans la tradition grecque pour que le passage soit omis tant dans le palimpseste que dans la grande majorité des témoins latins comme dans de bons témoins grecs ? Ou bien faut-il imaginer que le passage a été étendu afin de rendre la scène encore plus claire et vivante en ajoutant une intervention de Pilate? L'hypothèse de l'addition peut être soutenue par l'antiquité du palimpseste, qui a la forme courte ; mais celle de l'omission est étayée par la convergence des versions, qui ont la forme longue.

Ici, les familles latines $\mathrm{A}$ et $\mathrm{C}$ et quelques témoins grecs présentent un texte semblable à celui du palimpseste et plus court qu'une autre forme attestée par ailleurs. Un phénomène semblable se manifeste au chapitre 1.2: les juifs demandent à Pilate de faire comparaître Jésus (audire eum), et Pilate appelle un messager qui fasse entrer le prévenu (Advocans Pilatus cursorem.... $)^{29}$. Entre ces deux moments se trouve en grec, dans les versions orientales, dans les manuscrits latins isolés ${ }^{30}$ et dans la famille latine B un échange entre

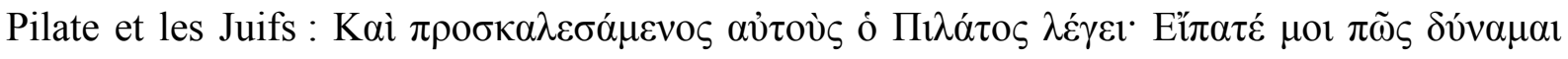

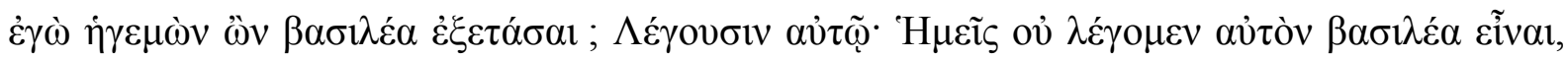
$\dot{\alpha} \lambda \lambda$ ' $\dot{\varepsilon} \alpha v \tau o ̀ v ~ \lambda \varepsilon ́ \gamma \varepsilon 1$, «Pilate les convoque et leur dit: Dites-moi comme je peux, moi qui suis gouverneur, soumettre un roi à l'interrogatoire? Ils lui disent : Nous, nous ne disons pas qu'il est roi, il le dit de lui »; et le texte poursuit : Tó $\varepsilon \varepsilon \pi \rho \circ \sigma \kappa \alpha \lambda \varepsilon \sigma \alpha ́ \mu \varepsilon v o \varsigma .$. On peut aisément supposer un saut du même au même entre la première et la seconde convocation par Pilate. Il est difficile de dire si c'est dans l'ancêtre latin ou dans le texte source grec que l'échange a été omis ; cet élément est intéressant pour les liens entre la famille latine A, le palimpseste et le texte grec, car il pourrait laisser penser que le palimpseste et la famille A ont un archétype commun.

\footnotetext{
${ }^{29}$ Seg. III, ch. 1.2, B8(138v).

${ }^{30}$ Il s'agit des manuscrits 59, 127, 129a, 299, 322 et 391.
} 
À peine élaborée, cette hypothèse doit être mise à mal, car un phénomène inverse se produit au chapitre 13.3. Là encore, le sens général de l'histoire n'est pas modifié par l'absence ou la présence des lignes en questions. Il s'agit d'un échange entre les gardes du tombeau et les Juifs, auxquels ils racontent l'apparition de l'ange aux femmes, en reprenant les termes du début de Mt 28. Le texte de la recension $\varphi$ donne le dialogue suivant :

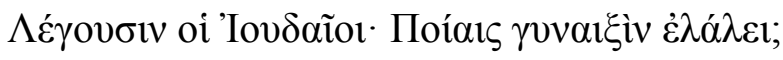

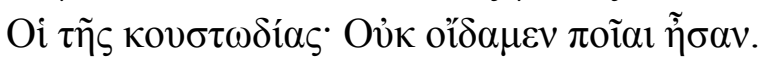

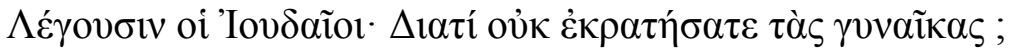

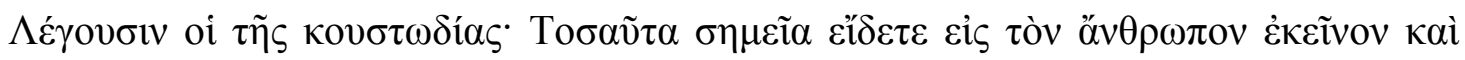

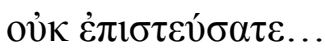

«Les juifs disent : À quelles femmes parlait-il?

Ceux de la garde : Nous ne savons pas qui elles étaient.

Les juifs disent : Pourquoi ne vous êtes-vous pas saisi des femmes?

Ceux de la garde disent : Vous avez vu tant de signes à propos de cet homme et vous n'avez pas cru, etc. »

Notons d'abord que les interlocuteurs des juifs sont désignés dans $\varphi$ comme oi $\tau \tilde{\eta} \varsigma$

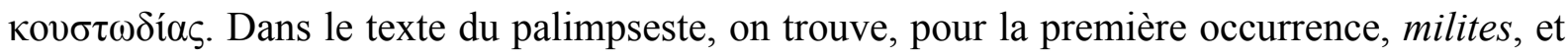
pour la seconde, custodes. Milites peut refléter le grec $\sigma \tau \rho \alpha \tau \iota \tilde{\omega} \tau \alpha \mathrm{l}$, qui se trouve dans le

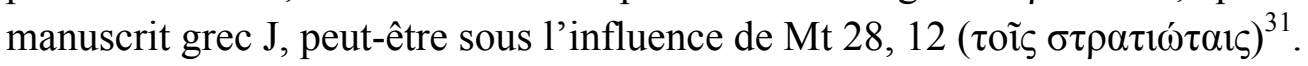

Ce jeu de dialogue, « Les Juifs dirent, les gardes dirent », peut rapidement entraîner un saut du même au même. Cela a peut-être été le cas; en effet, on a dans le palimpseste, à cet endroit :

\section{Dicunt Iudaei : Quibus mulieribus loquebatur?}

Dicunt milites : Nescimus que erant.

Dicunt Iudaei : Quae ora fuit?

Dicunt custodes : Media nocte.

Dicunt Iudaei : Quare non tenuistis mulieres?

Dicunt custodes Iudeis $<\ldots>^{32}$

L'échange supplémentaire, «Les juifs disent : Quelle heure était-il ? Les gardiens disent : Le milieu de la nuit», situe sinon le moment de la résurrection, du moins le moment de l'apparition de l'ange. Ces répliques se trouvent aussi dans les manuscrits inclassables grecs, $\mathrm{E}, \mathrm{B}$, et $\mathrm{N}$, ainsi que dans les versions arménienne, copte, géorgienne et syriaque du texte. Sur la base des trois manuscrits grecs, on peut ainsi reconstituer le texte source du palimpseste pour ces lignes :

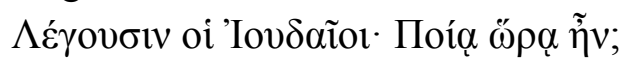

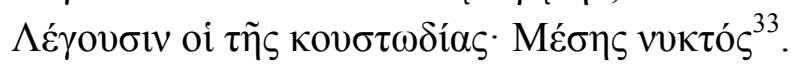

Ce texte est identique à celui du manuscrit grec B (les deux autres manuscrits présentent des variantes de détail) mais les lignes qui précèdent y sont différentes du texte fourni par le palimpseste : on ne peut donc pas dire pour autant que le texte source du palimpseste soit identifiable au manuscrit B.

\footnotetext{
${ }^{31}$ Les autres désignations des gardes dans cette péricope sont oi $\tau \eta \rho 0 \tilde{v} \tau \varepsilon \varsigma(\mathrm{Mt} 24,8)$ et $\tau 1 v \varepsilon \varsigma \tau \tilde{\eta} \varsigma$ $\kappa o v \sigma \tau \omega \delta i ́ \alpha \varsigma$ (Mt 28, 11).

${ }^{32}$ Seg. XIII, ch. 13.3, H3(162r).

${ }^{33}$ Tous les termes sont attestés par E, B et N, avec des variantes de détail dans N.
} 
Parmi les manuscrits latins, ceux qui transmettent cet échange de réplique sont minoritaires (il s'agit uniquement de la famille latine B et des manuscrits 127 et 129a, qui sont particuliers) ; la famille A l'omet. On a donc perdu la relation de proximité que les deux exemples précédents avaient semblé établir entre le palimpseste et les manuscrits de la famille A.

L'omission de la mention du « milieu de la nuit » survient donc après la traduction du texte dans les langues orientales et latine, qui l'attestent encore, mais assez tôt pour que toute la principale famille latine la reflète. Pour ne s'appuyer que sur les indications données par les manuscrits latins, elle se situe donc entre le $\mathrm{V}^{\mathrm{e}}$ siècle, époque du palimpseste, et le $\mathrm{IX}$ siècle, date des plus anciens manuscrits latins de la famille A.

Certes, le même phénomène peut survenir indépendamment en grec et en latin. Cela peut être possible pour un saut du même au même; cela peut l'être aussi s'il s'agit d'une omission consciente. Ce qui pourrait soutenir cette dernière hypothèse, c'est que l'enjeu derrière ces répliques est le moment de la résurrection - si l'on associe la résurrection à l'apparition de l'ange roulant la pierre. En effet, au début du chapitre 28 de Matthieu, cette apparition de l'ange est située par le narrateur avec une formule qui a suscité beaucoup de commentaires de la part des exégètes anciens et modernes, ỏ $\psi \grave{\varepsilon} \delta \dot{\varepsilon} \sigma \alpha b b a ́ \tau \omega v, \tau \tilde{n}$

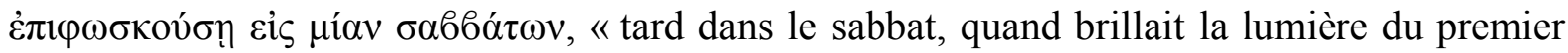
jour de la semaine ». Peut-être la disparition de l'échange entre les juifs et les gardes dans plusieurs familles des Actes de Pilate (majeure partie des manuscrits grecs, principales familles latines) est-elle une tentative d'orthodoxisation du texte des Actes de Pilate, afin d'éviter, autant que possible, la contradiction avec le récit canonique. On pourrait alors considérer que les traductions réalisées à date ancienne - les versions et les premiers témoins latins - auraient conservé le texte original des Actes de Pilate, alors que les formes plus tardives du texte, grecques comme latines, reflèteraient une tradition plus orthodoxe. Pour le latin, cette hypothèse serait à approfondir par l'étude des relations entre le palimpseste et la famille latine A.

B. Le palimpseste parmi les témoins d'un texte rarement attesté en grec

Quand Nicodème veut prendre la parole pour ouvrir la défense de Jésus, il demande à pouvoir prononcer «quelques paroles»-paucos sermones ${ }^{34}$ dans le palimpseste. On lit

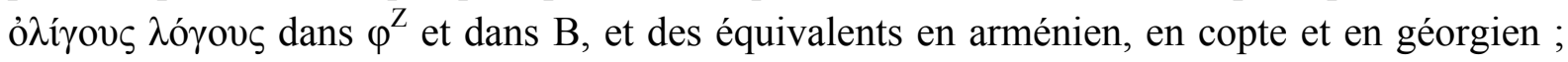
toute la tradition latine reflète cette leçon, et le syriaque est proche. En revanche, tous les autres manuscrits grecs ont $\kappa \alpha \theta \alpha \rho o v ̀ s ~ \lambda o ́ \gamma o v \varsigma$. Le passage de l'un à l'autre en grec serait difficile à expliquer par une confusion de copiste : sans doute faut-il faire l'hypothèse d'un remaniement du texte. L'antiquité du palimpseste vient soutenir l'attestation dans les versions orientales pour amener à considérer que la leçon « quelques paroles » est originale ; toutefois, c'est une lectio facilior.

\footnotetext{
${ }^{34}$ Seg. VII, ch. 5.1, D7(161v).
} 
C. Un texte attesté en latin mais absent de tous les manuscrits grecs conservés

Après le cas des formes grecques parfois attestées et rarement attestées parmi les témoins grecs conservés, on peut étudier des passages qui n'ont pas de parallèle dans une forme existante du grec.

Au chapitre 3.2, le célèbre entretien johannique de Jésus avec Pilate sur la question de la vérité est complété d'une manière originale par l'auteur des Actes de Pilate. Jésus dit à Pilate : Int [e]nde uerita $[t]<e m><$ dicentes $>^{35}<$ in terr $>a^{36}$, «Regarde ceux qui disent la vérité sur la terre ». Dans la plupart des manuscrits grecs qui ont un texte approchant, on lit

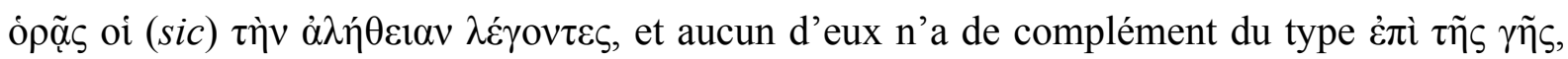
alors même que in terra est attesté dans l'ensemble des manuscrits latins des familles A et C. Il faut donc en conclure que ces familles, comme le palimpseste, dépendent d'une forme textuelle qui n'a pas survécu en grec. Comme il n'y a pas non plus d'équivalent de in terra dans l'ensemble des versions orientales, il pourrait être envisageable que l'expression appartienne à un archétype latin ; mais pour l'instant la dépendance de l'ensemble des manuscrits des familles $\mathrm{A}$ et $\mathrm{C}$ à un archétype latin n'est pas établie. L'insertion de l'expression in terra vient vraisembablement de ce que l'expression se trouve de toute façon à la fin de la phrase, «jugés par ceux qui ont le pouvoir sur la terre »: il s'agit d'une duplication dont on ne peut dire si elle trouve son origine en grec ou en latin.

Dans un autre cas, c'est la formulation elle-même qui est à étudier. Au chapitre 6.1, un témoin demande à prendre la parole, et Pilate 1'y encourage : Quod uis dicere dic ${ }^{37}$, « Ce que tu veux dire, dis-le ». Or aucun des manuscrits grecs conservés des Actes de Pilate ne contient d'équivalent exact de cette phrase. La plupart des manuscrits ont l'impératif final, ei $\pi \dot{\varepsilon}$, mais

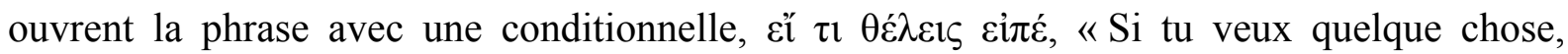
parle $»\left(\varphi^{\mathrm{GC}}, \mathrm{E}, \mathrm{N}\right.$; reformulé dans $\left.\varphi^{\mathrm{FXLZ}}\right)$. Le texte de la famille grecque $\chi$ est une

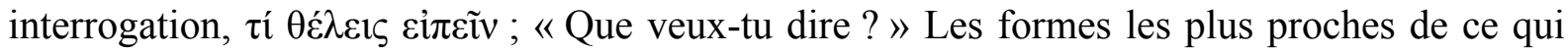
pourrait être le texte source du palimpseste sont dans les manuscrits inclassables B, cí $\tau$ l

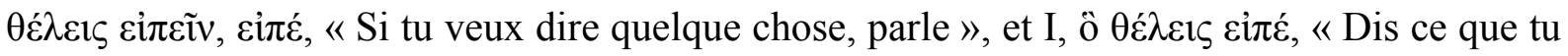
veux ». Il est bien évident que la répétition du verbe «dire» à des formes visuellement proches, $\varepsilon i \pi \varepsilon i v v$ et $\varepsilon i \pi \varepsilon$, a dû interférer dans la transmission de cette phrase. Le texte source du

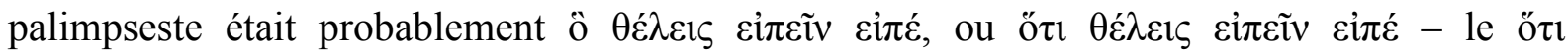
permettrait d'expliquer, surtout en onciale, le cỉ $\tau$. En grec, la disparition d'une des deux formes du verbe « dire » a pu entraîner la reformulation, par exemple sous forme de question,

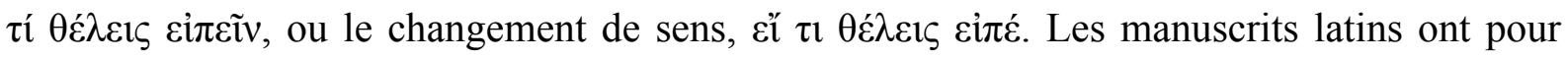
l'essentiel Quid uis dicere? Dic (familles A et C ; on trouve ponctuellement quod) et Loquere quod uis (famille B). Le palimpseste reflète visiblement un texte grec perdu, texte grec qui, ici, pourrait être à la source de toutes les formes conservées.

Ailleurs, le palimpseste est le seul témoin d'une évolution du texte grec probablement issue d'une mélecture. Au chapitre 16.1.3, les Juifs se rassemblent et discutent des témoignages qu'ils ont reçus sur Jésus. Un certain Lévi, désigné comme « didascale » dans le

\footnotetext{
${ }^{35}$ La reconstitution se fonde sur l'attestation dans certains manuscrits de la famille latine B $(160,369,387)$.

${ }^{36}$ Seg. V, ch. 3.2, C7(143r).

${ }^{37}$ Seg. VIII, ch. 6.1, E4(139v).
} 
texte grec, rapporte l'épisode au temple avec Syméon que nous avons déjà évoqué (Lc 2). Le texte grec de $\varphi$ enchaîne alors abruptement :

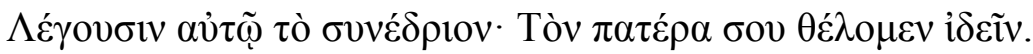

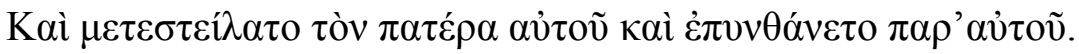

« Le conseil lui dit ${ }^{38}$ : Nous voulons voir ton père.

Et il envoya ${ }^{39}$ chercher son père et s'enquit auprès de lui. »

En revanche, le texte du palimpseste insère un dialogue entre la fin de la citation de Luc et la référence au père de Lévi :

Dicunt didascali et leuitae : Haec ista quomodo audisti?

Dicit Leui : Non scitis quoniam ab ipso didici legem?

Dicunt ipsi de concilio : Patrem tuum uolumus uidere.

Et scrutati sunt patrem eius et didicerunt ab eo... ${ }^{40}$

«Les didascales et les lévites disent : Ces paroles-là, comment les as-tu entendues?

Lévi dit : Vous ne savez pas que c'est de lui que j'ai appris la loi ?

Ceux du conseil disent : Nous voulons voir ton père.

Et ils recherchèrent son père et ils apprirent de lui...»

Cet échange et la précision apportée par le dénommé Lévi ne se trouvent donc pas dans le texte de $\varphi$, mais ils sont présents dans les versions orientales des Actes de Pilate ainsi que dans les manuscrits grecs E, B, N, et dans la famille $\chi$. Cependant, ici encore, aucun manuscrit grec ne reflète exactement le palimpseste. Ainsi, le sujet du premier dicunt - didascali et leuitae - ne se trouve dans aucun des témoins, ni grecs ni orientaux; après le verbe «ils dirent », les manuscrits grecs E, B, N, et la famille $\chi$ ont $\tau \tilde{\omega} \delta \delta \delta \alpha \sigma \kappa \alpha ́ \lambda \omega \Lambda$ $\Lambda \varepsilon v i ́$, ce qui donnerait en latin didascalo Leui. Or la plupart des manuscrits latins qui transmettent ce passage ont le pluriel didascali comme sujet de dicunt; en outre, des manuscrits de la famille latine B ont aussi des traces d'un ad Leui ${ }^{41}$, et deux manuscrits latins apparentés transmettent une leçon proche, Dicunt didascoli ad Leui : Tu quomodo ista audisti ${ }^{42}$, où Lévi est comme en grec le complément d'attribution.

On constate donc deux phénomènes. Le premier est l'omission du passage dans une partie de la tradition grecque (recension $\varphi$ ); l'inattention semble la meilleure explication : un échange a disparu par saut du même au même, mais le passage reste attesté par une autre partie de la tradition grecque et par les versions orientales et latines. Le second phénomène est caractéristique de la tradition latine: non seulement le datif $\delta 1 \delta \alpha \sigma \kappa \alpha ́ \lambda \omega$ est devenu le nominatif pluriel didascali, mais le nom propre $\Lambda$ cví est devenu leuitae, au nominatif pluriel. Sans doute s'agit-il d'une erreur de lecture en onciale: l'ancêtre du palimpseste et des manuscrits de la famille B avait sans doute la leçon ad Leui Tu..., conservée par deux des manuscrits latins (cf. supra). Le passage du datif singulier $\delta 1 \delta \alpha \sigma \kappa \alpha ́ \lambda \omega$ ou didascalo au nominatif pluriel $\delta ı \delta \alpha ́ \sigma \kappa \alpha \lambda \mathrm{ol}$ ou didascali a pu se produire en grec comme en latin : il n'est

\footnotetext{
${ }^{38}$ En grec, l'accord du verbe est fait selon le sens (pluriel).

${ }^{39}$ Ici il est vraisemblable que le sujet est toujours « le conseil ».

${ }^{40}$ Seg. XVII, ch. 16.1.3, J6(125v)- J7(164r).

${ }^{41} 284,336$ et 177 a (famille B1), avec des aléas de syntaxe.

42127 et 129 a.
} 
pas attesté dans les manuscrits grecs conservés. Le passage de Leui Tu à leuitae, en revanche, est propre au latin, et est facilité par la mention des lévites ailleurs dans le texte ${ }^{43}$.

Enfin, parmi les passages attestés en latin mais absents de tous les manuscrits grecs, doit être mentionné un cas de figure particulier qui est lié au phénomène même de la traduction de grec en latin. En effet, la préface des Actes de Pilate se termine dans le palimpseste par une invitation à faire mémoire et à prier pour celui qui est présenté comme le traducteur du texte d'hébreu en grec. Elle s'adresse à « vous tous qui copiez dans des volumes grecs ou latins » (Omnes ergo ... qui transfertis in aliis codicibus seu in grecis uel latinis ${ }^{44}$ ). Une formule semblable apparaît dans les deux seuls autres manuscrits qui ont cette prière (59

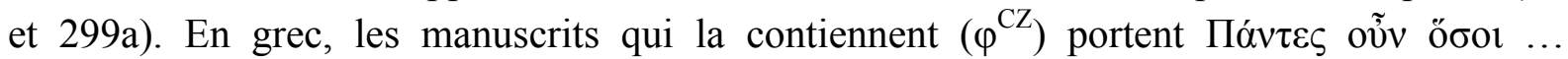

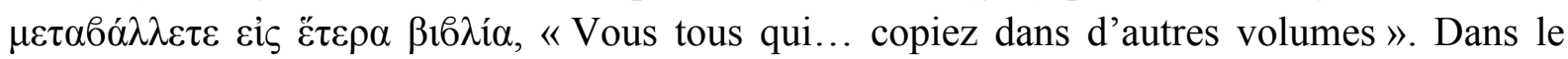
texte original grec, il ne peut bien sûr pas y avoir mention de l'alternative entre le grec et le latin ; c'est une innovation du traducteur, la seule peut-être qui soit évidente ${ }^{45}$.

Les six siècles qui séparent le premier témoin des Actes de Pilate des plus anciens manuscrits grecs ont permis l'évolution du texte grec vers différentes familles. Le texte du palimpseste correspond-il pour autant à l'archétype des manuscrits grecs ? Oui, par certains aspects : il reflète des formes qui peuvent appartenir indifféremment à l'un ou l'autre des manuscrits ou des groupes de manuscrits ; la comparaison avec les versions montre qu'il peut parfois entretenir une plus grande proximité avec des témoins issus des traductions anciennes qu'avec les manuscrits grecs. Mais les traductions dans les langues orientales ont elles aussi connu des évolutions et le texte du palimpseste n'est pas non plus particulièrement proche de l'une ou l'autre des versions. En outre, un certain nombre de passages font difficulté et font à s'interroger sur les relations qu'entretenait le texte source grec du palimpseste avec l'ensemble de la tradition des Actes de Pilate telle qu'on la connaît aujourd'hui.

\section{La traduction des citations bibliques, une ouvre originale}

Pour finir, on peut étudier un cas de traduction particulier, celui des citations bibliques. Toutes celles du texte mériteraient de retenir l'attention, car les choix de traduction pour les citations néotestamentaires dans le palimpseste sont originaux ${ }^{46}$ : les versets ne reprennent pas

\footnotetext{
${ }^{43}$ Quelques remarques sur le reste du passage : le haec ista peut s'expliquer par un usage pléonastique, attesté dans le TLL (vol. VI, c. 2743, 1. 11) ou refléter de quelque manière le oú présent après le $\tau \alpha \tilde{\tau} \tau \alpha$ dans les

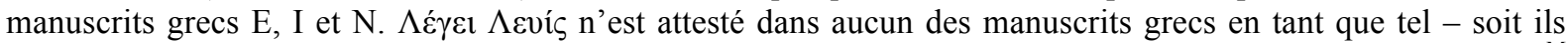

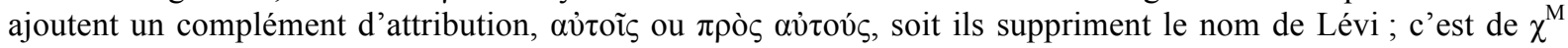
que le texte du palimpseste est le plus proche ( $\lambda \dot{\gamma} \gamma \varepsilon \imath$ ó $\Lambda \varepsilon v i \varsigma \varsigma$ ö $\imath$ ). La réponse de Lévi, en revanche, suit les manuscrits E, B et N. Enfin, la formule désignant l'interlocuteur de la ligne suivante, ipsi de concilio, n'a pas

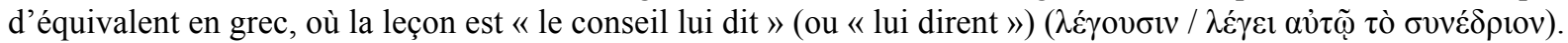
En revanche, le géorgien a une formule comme « des membres de l'assemblée lui dirent ». Il est donc possible qu'il y ait eu dans le texte source un pronom.

${ }^{44}$ Seg. I, préface, A6(173rv)

${ }^{45}$ Un autre ajout propre au texte du palimpseste, mais non caractéristique du texte latin, est l'expression $D e i$ Sabbatum (seg. III, ch. 1.1, B3[150r]), où l'ensemble des autres témoins, toutes langues confondues, a uniquement la mention du Sabbat.

${ }^{46}$ Mt 26,61; Mc 16,15; Mc 16,16; Lc 2,34; Lc 2,35; Lc 23,4 ; Lc 23,35; Lc 23,36; Lc 23,37; Lc 23,38 ; Lc 23,39; Lc 23,40; Jn 18,30; Jn 18,31. Exception : Jn 18,38.
} 
de forme Vetus Latina ou Vulgate connues, et ce pour la quasi-totalité de la vingtaine de versets cités littéralement; comme ce n'est pas le lieu ici d'évaluer le texte à l'intérieur de la tradition latine, je n'en donne que deux exemples, qui, outre l'originalité de leur forme latine, ont en commun de laisser planer un doute sur la forme du texte grec qui a pu servir à la traduction.

Au chapitre 14.1, des témoins racontent qu'ils ont vu Jésus parler avec ses disciples sur une montagne, une scène fragmentaire dans le palimpseste mais qui précède dans les Actes de Pilate le récit de l'Ascension. Est alors mis dans la bouche de Jésus un verset de la finale actuelle de Marc $(16,15)$ : Euntes in omnem saeculum adnuntiate omnia uniuersae

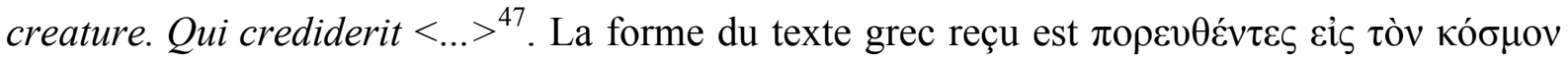

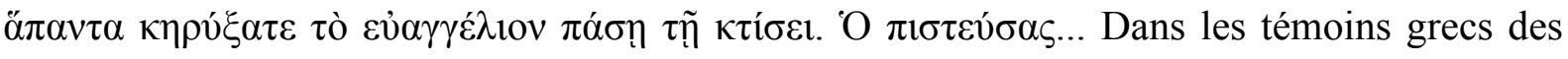
Actes de Pilate, le texte est relativement stable, si ce n'est que le complément d'objet, $\tau$ ò

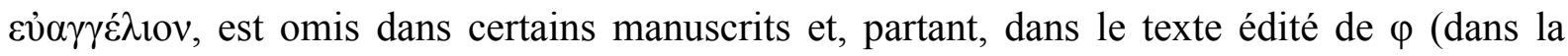
famille $\chi$, la phrase est réécrite).

Trois éléments sont curieux dans la traduction proposée dans le palimpseste. Tout d'abord, le groupe in omnem saeculum n'apparaît dans aucun des manuscrits latins de

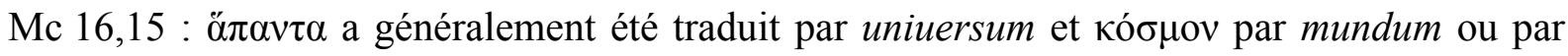
orbem ; mais il s'agit là de l'originalité du texte du palimpseste à l'intérieur de la tradition latine, originalité qui ne permet pas de supposer un texte source grec différent du texte reçu.

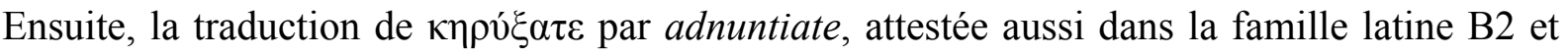
dans les manuscrits 127 et 129a, est originale car la traduction latine habituelle est praedicate, terme retenu dans les familles latines A et B1. Enfin, le complément de ce verbe est $\tau$ ò

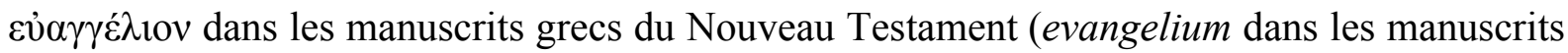
latins), et dans les manuscrits grecs $\varphi^{\mathrm{FXH}}$ et $\mathrm{E}$ (et $\chi$ ) des Actes de Pilate. En revanche, les manuscrits $\varphi^{\mathrm{GYCZ}}$, de même que $\mathrm{I}, \mathrm{B}$ et $\mathrm{N}$ n'ont pas ce complément, et n'ont rien qui le remplacent. Dans le palimpseste, omnia est présent comme complément du verbe principal :

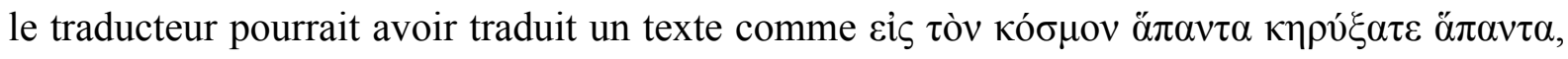
avec une curieuse répétition; il pourrait aussi avoir traduit deux fois ö $\pi \alpha v \tau \alpha$ dans une formule

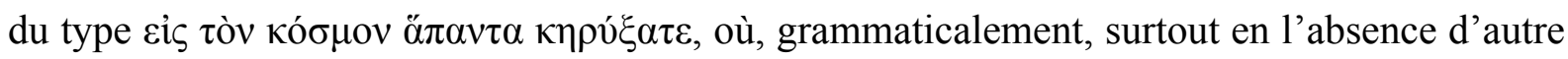

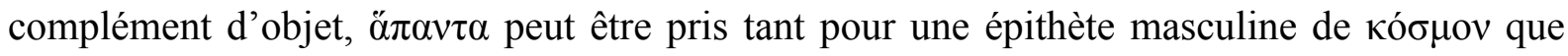
pour le complément au neutre pluriel de $\kappa \eta \rho v ́ \xi \alpha \tau \varepsilon$. On peut aussi s'interroger sur le choix de la traduction du verbe par adnuntiate, dont le radical évoque la "nouvelle » présente dans

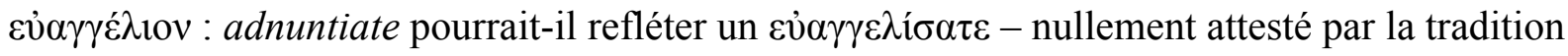

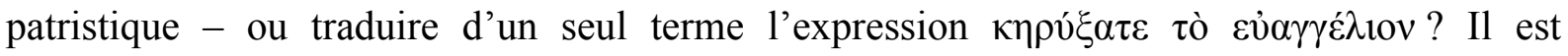
impossible de déterminer quelle était la forme du texte source grec du palimpseste à cet endroit.

Une autre citation, présente à deux reprises dans le texte, a déjà retenu notre attention pour son originalité. Il s'agit des paroles adressées par Syméon à la Vierge dans l'épisode de la présentation au Temple, au début de l'évangile de Luc. Dans le débat entre les juifs à la fin des Actes de Pilate est cité Lc 2,34-35 : Iste iacet in ruina et resurrectione mortuorum in Israhel et signum contradiç[tum]. Et tuam animam consumet romphea [qu]omodo reuelentur

\footnotetext{
${ }^{47}$ Seg. XV, ch. 14.1, I4(168v).
} 
de multorum cordibus cogitationes ${ }^{48}$, "Celui-ci est là pour la ruine et la résurrection des morts en Israël et comme signe contredit. Et ton âme, une épée la consumera, comme seront révélées les pensées au sujet des cœurs de beaucoup ». Le premier verset est cité de nouveau quelques lignes plus loin : Ecce iste iacet in ruina et resurrectio multorum Israhel et in signum contradictum est ${ }^{49}$ (ce verset paraît intraduisible mot à mot). Pour mémoire, le texte

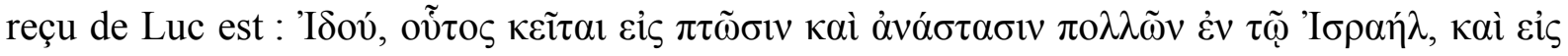

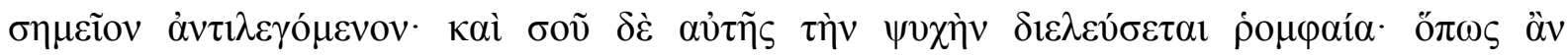

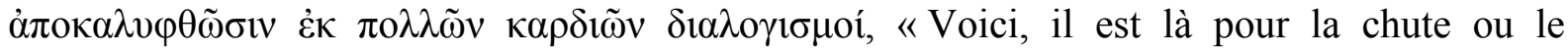
relèvement de beaucoup en Israël et pour être un signe contesté. Et toi-même, un glaive te transpercera l'âme ; ainsi seront dévoilés les débats de bien des cœurs » (traduction Segond).

Plusieurs points de traduction sont originaux. Les particularités que sont romphea et contradic [tum] ont déjà été évoquées. S'ajoute dans la deuxième citation la présence de est qui semble avec contradictum former un parfait. Est-ce une correction de copiste dérouté ? c'est vraisemblable. C'est sans doute aussi un copiste qui a oublié le in devant Israhel dans la deuxième occurrence et qui a utilisé le nominatif resurrectio.

Pour le choix du vocabulaire, iste, iacet et quomodo ne sont jamais retenus par les manuscrits bibliques latines, qui leur préfèrent hic, positus est et ut (alors même que la

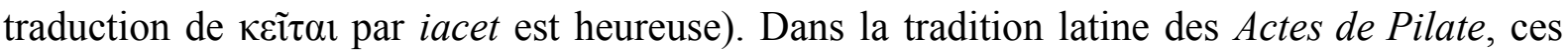
termes n'apparaissent que dans deux manuscrits ${ }^{50}$. La variante mortuorum est aussi inattendue, mais c'est une erreur du copiste due à l'association avec resurrectione ${ }^{51}$. La

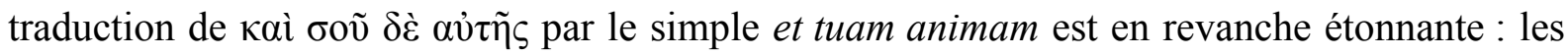
traductions latines du verset ne comportent pas toujours d'équivalent pour $\delta \dot{\varepsilon}$, mais toutes marquent $\alpha$ v่๊ก̃ par ipsius. Il est curieux que le palimpseste omette cette insistance.

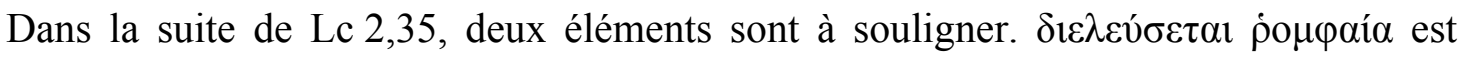
traduit par consumet romphea; or consumet exprime une image qui n'est pas dans $\delta 1 \varepsilon \lambda \varepsilon v ́ \sigma \varepsilon \tau \alpha$. Tous les manuscrits latins du Nouveau Testament ont ici une forme de pertranseo, qui reflète mieux le texte grec. Il est possible que l'image de la consumation soit

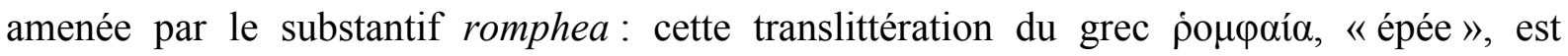
attestée en latin par des témoins patristiques faisant allusion à Gn 3,24 et décrivant l'arme des chérubins gardant le paradis comme une flammea romphea, " une épée flamboyante ${ }^{52}$. L'usage du verbe consumere serait-il une allusion à l'association entre l'épée et le feu ?

Enfin, dans l'expression de multorum cordibus cogitationes, l'usage du génitif multorum comme complément du nom cordibus est propre au palimpseste. L'ensemble des manuscrits du Nouveau Testament suit le grec et donne à l'adjectif le même cas que le substantif, comme en grec (multorum cordium ou ex / de multis cordibus). Le choix de la traduction de $\dot{\varepsilon} \kappa$ par de, partagé pour ce verset avec un seul manuscrit de la Vetus Latina, se retrouve à cinq reprises dans le palimpseste.

La traduction des versets Mc 16,15 et Lc 2,34-35 insérés dans les Actes de Pilate latins du palimpseste est unique. Elle reflète des choix originaux qui sont des hapax dans la tradition

\footnotetext{
${ }^{48}$ Seg. XVII, ch. 16.1.2, J5(125rv).

${ }^{49}$ Seg. XVIII, ch. 16.3.2, K3(130rv).

${ }^{50} \mathrm{Il}$ s'agit toujours des manuscrits 127 et 129 a.

${ }^{51}$ M. DespinEUX, «Une version latine palimpseste du $\mathrm{v}^{\mathrm{e}}$ siècle de l'Évangile de Nicodème (Vienne, ÖNB MS 563), Scriptorium 42 (1988), p. 176-183, p. 182.

${ }_{52}$ Cf. e.g. Jérôme, Commentaire sur Amos 3, 6 ; Augustin, Rétractations 2, 24.
} 
latine, parmi les manuscrits du Nouveau Testament et des Actes de Pilate comme parmi les témoins patristiques. Les autres citations scripturaires du texte sont à l'avenant. Ces choix lexicaux et grammaticaux n'impliquent pas un texte source différent du texte grec reçu; en revanche, ils sont assez étonnants pour que l'on puisse s'interroger sur l'identité du traducteur. Qui, au $\mathrm{V}^{\mathrm{e}}$ siècle, pouvait encore avoir une telle liberté par rapport au texte biblique? S'agirait-il d'un traducteur qui n'avait aucune pratique régulière du texte biblique latin et ignorait tout des formulations habituelles?

\section{Conclusion}

Le rapprochement du texte latin des Actes de Pilate transmis par le palimpseste de Vienne avec l'ensemble des témoins du texte grec des Actes de Pilate ne permet pas de faire ressortir l'un ou l'autre manuscrit grec comme étant proche de celui qui a servi au traducteur de l'ancêtre du palimpseste. En revanche, l'examen attentif du texte transmis par le palimpseste permet de redonner sa juste place au plus ancien témoin manuscrit des Actes de Pilate. Le premier contexte dans lequel il s'insère est celui des traductions du grec en latin au $\mathrm{V}^{\mathrm{e}}$ siècle, et particulièrement des traductions bibliques : le texte latin des Actes de Pilate dans le palimpseste révèle un souci de proximité avec la langue de départ, marqué par la transcription de mots rares et le calque de certaines tournures. Jointe à ce sentiment d'étrangeté pour les termes peu fréquents en grec, l'originalité dans la traduction des citations scripturaires insérées dans le récit pourrait trahir un manque de familiarité avec le texte biblique, et très certainement avec la forme latine de celui-ci. S'agirait-il d'un traducteur grec n'ayant aucune pratique de la version latine des Écritures ? Cela pourrait expliquer certaines maladresses du latin.

Le second contexte dans lequel cette forme latine des Actes de Pilate est à étudier est celui des différentes formes du récit transmises en grec, en latin et dans les langues orientales. La relation du texte du palimpseste avec le texte grec montre que les manuscrits grecs présentent une forme retouchée, qui s'éloigne des points d'accord entre le texte du palimpseste et les versions orientales. Avec les formes latines, et notamment avec la famille latine $\mathrm{A}$, le texte du palimpseste oscille de la proximité à l'éloignement, selon les passages ; on pourrait faire l'hypothèse d'un archétype commun au palimpseste et à la famille latine $\mathrm{A}$, en ajoutant que l'ancêtre propre à la famille A aurait subi certaines modifications, peut-être par souci d'orthodoxiser des éléments de la narration. Enfin, le texte des Actes de Pilate dans le palimpseste comporte un certain nombre d'accidents propres ; comme tout témoin, il n'est pas entièrement fiable, et, si son antiquité fait sa valeur, elle ne lui confère pas l'autorité. 


\section{Appendice : références des manuscrits grecs et latins}

\section{Témoins manuscrits grecs directs des Actes de Pilate groupés par familles ${ }^{53}$}

Famille $\varphi$

C Paris, Bibliothèque nationale de France, MS gr. 770 (olim Colbert 2493; Regius 2356.1.1) (a. 1315)

F Milano, Biblioteca Ambrosiana, MS gr. 192 (C92 sup., olim N 155) (s. xiv/1)

G Milano, Biblioteca Ambrosiana, MS gr. 4 (A 56 sup.) (s. xvi)

$\mathrm{H} \quad$ London, British Library, MS Harley gr. 5639 (s. xiv, xvi)

K London, British Library, MS Harley gr. 5636 (s. xvi)

L Meteōra, Monē Metamorphōseōs, MS 549 (s. xiv-xv)

X Meteōra, Monē Rousanou, MS Hagia Trias 14 (Rousanou 12) (s. Xv-xvi)

Y Meteōra, Monē Rousanou, MS Hagia Trias 90 (s. xvi)

Z Roma, Biblioteca nazionale centrale, MS gr. 20 (s. xv)

Famille $\chi$

A München, Bayerische Staatsbibliothek, MS gr. 192 (s. xiv)

M Hagion Oros, Monē Megistēs Lauras, MS K 81 (a. 1368)

O Athēnai, Ethnikē Bibliothēkē tēs Hellados, MS 2187 (s. xv)

Q Hagion Oros, Monē Docheiariou, MS 114 (s. xvi)

W Hagion Oros, Monē Batopediou, MS 776 (s. xviii)

Manuscrits inclassables

B München, Bayerische Staatsbibliothek, MS gr. 276 (s. xii)

E Paris, Bibliothèque nationale de France, MS gr. 929 (olim Colbert 5104) (s. Xv)

I München, Bayerische Staatsbibliothek, MS gr. 524 (s. xiv)

J Andros, Monē Zoōdochos pēgēs ē Hagias, MS 46 (s. xv)

N Hagion Oros, Monē Megistēs Lauras, MS $\Lambda 117$ (s. xvi)

\section{Témoins manuscrits latins des Actes de Pilate cités dans cet article ${ }^{54}$}

59 Cambridge, University Library, MS Ff.VI.54 (s. xiv)

127 Kraków, Biblioteka Jagiellońska, MS 1509 (s. xv ex.)

129 Kraków, Biblioteka Jagiellońska, MS 2724

129a ${ }^{55}$ Kraków, Polska Akademia Umiejętności, MS 1713 (1471)

133 Laon, Bibliothèque municipale, MS 265 (s. ix)

158 London, British Library, MS Royal 5 E. XIII (s. ix)

177a Montecassino, Archivio dell'Abbazzia, Cod. 117 GG (s. xi-xii)

207 München, Bayerische Staatsbibliothek, Clm 29275 (s. ix)

213 Olomouc, Kapitulní knihovna, CO 407 (s. xv in.)

284 Paris, Bibliothèque nationale de France, MS lat. 14864 (s. xii ex.)

299 Praha, Knihovna metropolitní kapituly, MS n. LIV (1478)

\footnotetext{
${ }^{53}$ Liste établie d'après les indications de C. FURRER, « La recension greque ancienne des Actes de Pilate », Apocrypha 21 (2010), p. 11-30 et, pour trois manuscrits découverts par la suite, dans C. FURRER et C. GuIGNARD, «Titre et prologue des Actes de Pilate : nouvelle lecture à partir d'une reconstitution d'un état ancien du texte », Apocrypha 24 (2013), p. 139-206, Appendice 4, p. 204-205.

${ }^{54}$ Les sigles sont ceux attribués par Z. IZYDORCZYK, Manuscripts of the Evangelium Nicodemi. A Census, coll. « Subsidia Mediaevalia » 21, Toronto, 1993.

${ }^{55}$ Ce manuscrit n'était pas connu au moment de la composition du Census.
} 
322 Praha, Státní knihovna, MS XX.A.7 (s. xiv-xv)

334 Saint-Omer, Bibliothèque municipale, MS 202 (s. ix)

336 Salzburg, Erzabtei St. Peter, MS a V 27 (s. xii/2)

391 Washington, D.C., Library of Congress, MS 95 (Faye and Bond 114) (s. xvi in.) 